\title{
The use of external within-person variance estimates to adjust nutrient intake distributions over time and across populations
}

\author{
Lisa Jahns ${ }^{1}$, Lenore Arab ${ }^{2}$, Alicia Carriquiry ${ }^{3}$ and Barry M Popkin ${ }^{4,5, *}$ \\ ${ }^{1}$ Department of Nutrition, University of Tennessee, Knoxville, TN, USA: ${ }^{2}$ Department of Epidemiology, School of \\ Public Health, University of North Carolina, Chapel Hill, NC, USA: ${ }^{3}$ Department of Statistics, lowa State University, \\ Ames, IA, USA: ${ }^{4}$ Department of Nutrition, School of Public Health, University of North Carolina, Chapel Hill, \\ NC, USA: ${ }^{5}$ Carolina Population Center, University of North Carolina at Chapel Hill, CB \#8120 University Square, \\ 123 W. Franklin Street, Chapel Hill, NC 27516-3997, USA
}

Submitted 9 June 2004: Accepted 17 August 2004

\begin{abstract}
Objective: To examine the utility of using external estimates of within-person variation to adjust usual nutrient intake distributions.

Design: Analyses of the prevalence of inadequate intake of an example nutrient by the Estimated Average Requirement (EAR) cut-point method using three different methods of statistical adjustment of the usual intake distribution of a single 24-hour recall in Russian children in 1996, using the Iowa State University method for adjustment of the distribution. First, adjusting the usual intake distribution with day 2 recalls from the same 1996 sample (the correct method); second, adjusting the distribution using external variance estimates derived from US children in 1996; and third, adjusting the distribution using external estimates derived from Russian children of the same age in 2000 . We also present prevalence estimates based on naïve statistical analysis of the unadjusted distribution of intakes.

Setting/subjects: Children drawn from the Russia Longitudinal Monitoring Survey in 1996 and 2000 and from the 1996 Continuing Survey of Food Intakes by Individuals. Results: When the EAR cut-point method is applied to a single recall, the resulting prevalence estimate in this study is inflated by 100-1300\%. When the intake distribution is adjusted using an external variance estimate, the prevalence estimate is much less biased, suggesting that any adjustment may give less biased estimates than no adjustment.

Conclusions: In moderately large samples, adjusting distributions with external estimates of variances results in more reliable prevalence estimates than using 1-day data.
\end{abstract}

Nutrient intakes are associated with health and with disease risk, and are used in public health to inform national nutrition policies regarding food assistance programmes, fortification needs and exposure to contaminants ${ }^{1,2}$.

The parameter of interest is not an individual's one-day intake, but their usual intake, defined as the long run average of daily intakes of a dietary component by an individual' ${ }^{3}$. In theory, if an individual's daily intake could be observed every day for a long period of time, say a year or two, the estimate would be a reliable estimate of the individual's usual intake ${ }^{4}$. The accuracy of assessment of adequate nutrient intake of population groups depends upon a reasonable estimate of usual nutrient intake distribution of that group, with accurate estimation of the intake percentiles in the population ${ }^{5-9}$. Dietary assessment, especially of children, is fraught with random and systematic error ${ }^{9,10}$. Short-term measurements such as 24-hour recalls, diet records and food diaries contain an additional variance component: the normal, day-to-day fluctuations in intake of free-living populations. This fluctuation is also known as within-person variation ${ }^{11}$. Significant within-person variability can obscure the true between-person variation that is of interest in distinguishing those groups with adequate or inadequate intake ${ }^{9,12}$.

One school of thought proposes that if at least two nonconsecutive or three consecutive recalls are collected on at least a sub-sample of the study group, the within-person variation can be calculated and its effect partially adjusted statistically ${ }^{8,12}$. The collection of additional days of recall, although desirable, may be cost-prohibitive, especially in sub-populations of interest. Collecting a 1-day recall would be least expensive and burdensome, but would contain no information about within-person variation. Due to cost and respondent burden, many national surveys, including the US Continuing Survey of Food 
Intakes by Individuals (CSFII) and the forthcoming integrated 'What We Eat in America-NHANES', collect two non-consecutive 1-day recalls ${ }^{6}$.

If only a single short-term recall is collected, the usual intake distribution of the group cannot be determined. The Third National Health and Nutrition Examination Survey (NHANESIII) 1989-1994, a large, nationally representative US study, collected a single 24-hour recall for approximately 30000 people and a second recall on 5\% of adults. The replicate was collected for the purpose of estimating within- and between-person variances for the estimation of nutrient intake distributions. For adults then, it is possible to calculate usual intake percentiles ${ }^{13}$. However, usual intake cannot be determined in NHANESIII data for most subpopulations (i.e. children), as repeat measures were not collected in this population. Other studies have erroneously reported inadequate group intakes using the North American Dietary Reference Intakes (DRIs), based upon an unadjusted single day's intake ${ }^{14,15}$.

In the case of a study where only a single short-term recall has been collected, the possibility of using an appropriate external estimate of within-person variability to analyse a subgroup of interest in relation to the DRIs has been suggested ${ }^{6,16-18}$. However, the issue of what may constitute an appropriate external estimate has not been discussed. If an estimate is used from a group with much higher or lower within-person variability than the target group, then the usual intake derived from applying the variance of a divergent group may be incorrect.

A second alternative would be using an estimate of within-person, day-to-day variation from the same population, but several years before or after the time period for which adequacy is estimated. Changes over time in the food supply and eating patterns could lead to differential changes in within- and between-person variation. These changes could seriously compromise results from cohort studies that collect multiple days of intake at the beginning of the study and then use those estimates of within-person variation to adjust intake data in subsequent rounds of data collected months or even years later, or to calibrate dietary intake data from successive food-frequency questionnaires.

The present study builds upon previous research wherein we demonstrated that within- and betweenperson variation in Russian and US children's diets, as estimated by 24-hour recall, varies substantially by age and sex group and between the two countries (Jahns L, Carriquiry A, Arab L, Mroz T, Popkin BM. Within- and between-person variability of nutrient intakes of Russian and US children differs by sex and age. Unpublished results). We hypothesised about the feasibility of using estimates of within-person variation from one nationally representative population subgroup (say, US children of some age and sex subgroup) to adjust the usual intake distribution of another group (in this case, Russian children of the same age and sex subgroup).
To our knowledge, no one has examined the usefulness of using appropriate external estimates of within-person variation to adjust usual nutrient intake distributions. We report and compare prevalence estimates of inadequate intake of one example nutrient, vitamin C, amongst Russian children in 1996. We chose vitamin C as a nutrient of public health interest that also has an Estimated Average Requirement (EAR). We apply the EAR cut-point approach using three different methods of statistical adjustment of the usual intake distribution of a single 24-hour recall. First, adjusting the usual intake distribution with day 2 recalls from the same 1996 sample (the correct method); second, adjusting the distribution using external variance estimates derived from US children in 1996; and third, adjusting the distribution using external estimates derived from Russian children of the same age in 2000. We also present prevalence estimates based on naïve statistical analysis of the unadjusted distribution of intakes.

\section{Participants and methods}

\section{Study population}

Data were derived from two studies: the Russia Longitudinal Monitoring Survey (RLMS) and the CSFII. The RLMS is an ongoing split-panel survey of health and economic change in the Russian Federation. Details of the design and collection of the study can be found elsewhere ${ }^{19,20}$. This study was approved by the Institutional Review Board at The University of North Carolina School of Public Health.

The CSFII is a series of cross-sectional, nationally representative surveys of the kinds and amounts of food eaten by Americans and has been described in detail elsewhere $^{21}$.

\section{Data collection}

Both studies collected 24-hour recalls. In 1994, 1995 and 1996, CSFII collected two interviewer-administered 24-hour recalls, using a multiple-pass approach. Children aged 12 years and over self-reported intake, while data for those under 12 were provided by a parent or caregiver. The final sample consists of 3869.0-13.9-year-old children with complete data interviewed in 1996 (CSFII 1996).

In 11 of the 12 RLMS data collection rounds, a single 24-hour recall was collected. In 1996 two non-consecutive recalls were collected for all individuals (RLMS 1996), and in 2000 a sub-sample of 250 children provided three consecutive 1-day recalls (RLMS 2000). Children aged 10 years and over self-reported intake, while data for those under 10 were provided by a parent or caregiver. Having multiple recalls at two time points allows us to compare potential time differences in within-person variation. The RLMS 1996 sample consists of 798 children with two complete recalls. The RLMS 2000 sample consists of 103 9.0-13.9-year-old children ( 45 girls and 58 boys) from the 
RLMS round 9 (conducted from October to December 2000), who provided three recalls.

Only nutrients contributed from food sources were considered in this analysis. Dietary supplement use in US children can be considerable ${ }^{22}$, but is very low among Russian children. Country-specific food composition tables (FCTs) were used to calculate nutrient values as the US food supply contains many more fortified foods than the Russian food supply.

\section{The EAR cut-point method}

The goal of the present analysis was to test the hypothesis that we can use external estimates of intra-individual variation to adjust usual intake distributions, leading to prevalence estimates of inadequate that are comparable to using the internal (correct) intra-individual variation. To assess the prevalence of nutrient inadequacy, we use the EAR cut-point methodology ${ }^{12}$. Using this method, the prevalence of inadequate nutrient intake in a particular age and sex group of individuals is the proportion of the group with intakes below the median requirement. The EARs are based upon risks of deficiency and, for some nutrients, chronic disease prevention ${ }^{23}$. The methods have

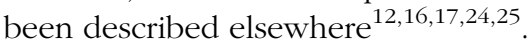

Major assumptions of this method include:

1. Intakes are accurately measured;

2. The actual prevalence of inadequate intake is neither particularly high nor low;

3. Estimated usual intakes are independent of estimated requirements (assumed to be true except for energy);

4. The distribution of requirements is not skewed (assumed to be true for all nutrients except iron);

5. The variability of intakes in the group is hypothesised to be greater than the variability of requirements (assumed to be true).

The EAR for vitamin C for 9-13-year-old girls and boys is $39 \mathrm{mg}^{23}$.

\section{Software for Intake Distribution Estimation (PC-SIDE)}

The personal computer version of Software for Intake Distribution Estimation (PC-SIDE) (version 1.0, 2003; available from the Department of Statistics, Iowa State University, Ames, IA, USA) and the supporting documentation were used to estimate usual intake distributions ${ }^{26,27}$. Details of the methodology, also known as the Iowa State University (ISU) method, are discussed in detail elsewhere, and are built upon methods proposed and developed by Nusser and colleagues, including one of the authors ${ }^{3,8,24}$. PC-SIDE software produces an empirical estimate of the usual nutrient intake of each EAR age and sex subgroup, estimates adjusted percentiles and calculates the prevalence of inadequate intake based upon the subgroup EAR cut-point method. Its use has been endorsed by Dwyer et al. ${ }^{6}$ and Hoffman et $a l^{28}$.

\section{Covariates}

Many individual-level factors affect the extent of a person's within-person variation in nutrient intake. For instance, if lower-income people were to have only sporadic access to vitamin-C-rich foods and higher-income people have constant access, then part of the within-person variation will be due to income. Researchers often control for sociodemographic factors in predictive models of nutrient intake. In this analysis, we control for poverty and urban residence in a general way, using country-specific measures of 'poverty' and 'urban' residence. These controls are not meant to create comparability between the samples, but rather to control for some of the larger differences within countries.

In the Russian sample, the poverty variable was based on the official Russian poverty index. This measure, developed by one of the authors in conjunction with other Russian officials and researchers, reflects the average cost of food items in a Russian food basket for low-income persons and is similar to the 'thrifty food plan' based on the US poverty line ${ }^{29}$. This household poverty index is derived as nominal household income/sum of all individual poverty lines in household, adjusted for household size. We dichotomise the variable to poverty $(0 / 1)=<100 \%$ of the poverty index. Details on the income and price measures can be found elsewhere ${ }^{30}$. The US poverty variable is dichotomised as poverty $(0 / 1)=<185 \%$ of the poverty level. This is the level at which a family becomes eligible for food assistance programmes in the USA. 'Urban' in both surveys refers to MSA designation 1 and 2.

\section{Statistical analysis}

Sociodemographic characteristics for 9-13-year-old girls and boys are presented as prevalence and mean \pm standard deviation (SD) for each sample (Table 1). Mean \pm SD values of vitamin $\mathrm{C}$ intake from food sources are given (Table 2).

The adjusted variance estimates from PC-SIDE for each sample group's transformed distribution of vitamin C intake were calculated (Table 3). We first weighted the CSFII 1996 and the RLMS 2000 groups to the same age and sex distribution as the RLMS 1996 group. For each sex within each sample group we calculated the usual intake distribution of vitamin $\mathrm{C}$ and the prevalence of inadequate intake (based upon the EAR cut-point method), using the standardised weights. In each of the six models we adjusted for the effects of day of week of the interview, interview sequence, poverty and urban residence. In the RLMS 2000 sample, we also adjusted for the correlation of intakes reported over consecutive days, using previously published figures ${ }^{31}$. From the outputs we abstracted the two numbers needed for the external variance adjustment. The prior estimate of the variance component ratio refers to the ratio of within- to between-person variation, and the prior estimate of fourth moment of measurement error 
Table 1 Demographics of 9-13-year-old children from the Russia Longitudinal Monitoring Survey (RLMS) and the US Continuing Survey of Food Intakes by Individuals (CSFII)

\begin{tabular}{lcccc}
\hline & Sample $(n)$ & Age (years), mean \pm SD & \% Below poverty level & \% Residing in urban centre \\
\hline Girls & & & & \\
RLMS 1996 & 402 & $11.0 \pm 1.4$ & 44.8 & 73.9 \\
CSFII 1996 & 176 & $10.9 \pm 1.4$ & 23.9 & 71.6 \\
RLMS 2000 & 45 & $11.0 \pm 1.6$ & 35.6 & 80.0 \\
Boys & & $10.9 \pm 1.4$ & 44.0 & 70.1 \\
RLMS 1996 & 371 & $10.8 \pm 1.3$ & 20.9 & 76.5 \\
CSFII 1996 & 196 & $10.7 \pm 1.5$ & 37.9 & 72.4 \\
RLMS 2000 & 58 & & & \\
\hline
\end{tabular}

SD - standard deviation.

Table 2 Estimated day and average intake of vitamin C $(\mathrm{mg})$ by sex for 9-13-year-old children from the Russia Longitudinal Monitoring Survey (RLMS) in 1996 and 2000*, and the US Continuing Survey of Food Intakes by Individuals (CSFII) in 1996

\begin{tabular}{lccc}
\hline Sample $(n)$ & $\begin{array}{c}\text { Day 1 24-hour recall, } \\
\text { mean } \pm \text { SD }\end{array}$ & $\begin{array}{c}\text { Second 24-hour recall, } \\
\text { mean } \pm \text { SD }\end{array}$ & $\begin{array}{c}\text { Average, } \\
\text { mean } \pm \text { SD }\end{array}$ \\
\hline Girls & & & \\
RLMS 1996 (402) & $59.6 \pm 51.7$ & $52.0 \pm 47.8$ & $54.8 \pm 50.0$ \\
CSFII 1996 (176) & $88.0 \pm 77.7$ & $82.9 \pm 66.0$ & $85.4 \pm 72.0$ \\
RLMS 2000 (45) & $59.6 \pm 44.8$ & $74.6 \pm 77.9$ & $65.2 \pm 61.3$ \\
Boys & & & \\
RLMS 1996 (371) & $54.0 \pm 47.5$ & $44.7 \pm 35.3$ & $49.4 \pm 42.0$ \\
CSFII 1996 (196) & $95.6 \pm 77.8$ & $90.0 \pm 75.5$ & $92.8 \pm 76.6$ \\
RLMS 2000 (58) & $52.8 \pm 44.7$ & $55.0 \pm 52.1$ & $54.6 \pm 46.5$ \\
\hline
\end{tabular}

SD - standard deviation.

*Three-day average.

distribution refers to the kurtosis, or flatness, of the distribution curve.

Next, to determine if the external variance estimates are different enough to cause meaningful changes in the observed prevalence of inadequate vitamin $\mathrm{C}$ intake, we used the EAR cut-point method, implemented using PC-SIDE. The external variance estimates from the CSFII 1996 and RLMS 2000 (Table 3) were used to adjust the usual intake of the RLMS 1996 children.

Specifically, to apply the external variance estimates, we opened the same dataset from the RLMS 1996 girls (then boys). First, we opened the 'Configuration' window, and

Table 3 Variance estimates* used to adjust RLMS 1996 usual intake distribution

\begin{tabular}{ccc}
\hline & $\begin{array}{c}\text { Prior estimate } \\
\text { of the within- to } \\
\text { between-person variance } \\
\text { component ratio }\end{array}$ & $\begin{array}{c}\text { Prior estimate of } \\
\text { fourth moment of } \\
\text { measurement error } \\
\text { distribution (kurtosis) }\end{array}$ \\
\hline 9-13-year-old girls & & \\
RLMS 1996 & 0.69034 & 3.5962 \\
CSFII 1996 & 0.60708 & 3.0680 \\
RLMS 2000 & 0.69113 & 3.4269 \\
9-13-year-old boys & & \\
RLMS 1996 & 0.54390 & 3.0000 \\
CSFII 1996 & 0.75414 & 4.4625 \\
RLMS 2000 & 0.61146 & 3.6090 \\
\hline
\end{tabular}

RLMS - Russia Longitudinal Monitoring Survey; CSFII - US Continuing Survey of Food Intakes by Individuals.

${ }^{*}$ All prior (external) estimates are obtained through PC-SIDE software. These are the external estimates used by PC-SIDE to adjust the distribution in Table 4 , and are estimated from data that have already been transformed into the normal scale. then the 'Parameters' tab. At the bottom is a box marked 'Prior estimated variance component ratio specified for each run?' which had to be left unchecked. Next, the two components of the external variance estimate were typed in the appropriate box. Finally, the last box is labelled 'Number of individuals associated with the prior variance component ratio (0 implies infinity)'. This box was set to zero. The model was then run again using the external variance estimates (Table 4).

We list the top food group sources of children's vitamin C intake for each sample group (Table 5).

Data management and descriptive statistics were performed using SAS and Stata software ${ }^{32,33}$.

\section{Results}

Sociodemographic characteristics for each sample are described in Table 1. The mean age of 9-13-year-old children was similar in all three samples and between girls and boys. Twice as many Russian as US children lived in poverty in 1996 ( 44.3 vs. 22.3\%), but the proportion of Russian children living in poverty declined between 1996 and 2000 ( 44.3 to $36.9 \%$ ). A high proportion of children in all samples resided in urban areas ( $>70 \%)$. Among the Russian children, slightly more children lived in urban areas in the 2000 sample than in the 1996 sample.

Russian children had considerably lower vitamin C intakes than US children (Table 2). Russian boys in 1996 had the lowest mean intake of vitamin C ( $49 \mathrm{mg})$, lower 
Table 4 Prevalence of inadequate vitamin $C$ intake by the Estimated Average Requirement cut-point method using internal and external variance estimates to adjust the usual intake distribution, 9-13-year-old children

\begin{tabular}{|c|c|c|c|c|}
\hline & $\begin{array}{c}\text { Adjusted } \\
\text { true value } \pm \mathrm{SE}\end{array}$ & Unadjusted $^{*}$ & $\begin{array}{l}\text { Adjusted with CSFII } \\
1996 \text { variance } \pm \text { SE }\end{array}$ & $\begin{array}{l}\text { Adjusted with RLMS } \\
2000 \text { variance } \pm \text { SE }\end{array}$ \\
\hline \multicolumn{5}{|l|}{ Girls } \\
\hline RLMS 1996 & $22.97 \pm 0.05$ & 45.15 & $27.30 \pm 0.04$ & $22.98 \pm 0.05$ \\
\hline CSFII 1996 & $10.93 \pm 0.05$ & 31.82 & - & - \\
\hline RLMS 2000 & $26.37 \pm 0.11$ & 41.48 & - & - \\
\hline \multicolumn{5}{|l|}{ Boys } \\
\hline RLMS 1996 & $35.83 \pm 0.03$ & 51.35 & $33.27 \pm 0.04$ & $32.56 \pm 0.04$ \\
\hline CSFII 1996 & $2.20 \pm 0.03$ & 26.53 & - & - \\
\hline RLMS 2000 & $29.19 \pm 0.09$ & 47.13 & - & - \\
\hline
\end{tabular}

SE - standard error; CSFII - US Continuing Survey of Food Intakes by Individuals; RLMS - Russia Longitudinal Monitoring Survey.

${ }^{*}$ Proportion with mean 1-day vitamin C intakes below $39 \mathrm{mg}$.

than both Russian girls and US children. The mean day 2 vitamin $\mathrm{C}$ intakes were lower than the day 1 intakes for all participants except the RLMS 2000 girls, who reported a much higher intake level on day 2.

The variance components obtained from PC-SIDE for each sample are shown in Table 3. The variance components are estimated from data that have already been transformed into the normal scale and the ratios should be interpreted as follows: in the transformed scale, the ratio represents the relative proportion of within- to between-person variance in intake. For example, in the case of Russian girls in 1996, 69\% of the total variation in daily intake of vitamin $\mathrm{C}$ can be attributed to withinperson variability and 31\% can be attributed to betweenperson variability.

It is difficult to determine how different the variance estimates are from each other, so we substituted the external estimates from the CSFII 1996 and the RLMS 2000 samples into the model for the RLMS 1996 sample.

Table 4 shows the prevalence of inadequate vitamin $\mathrm{C}$ intake in each sample using the EAR cut-point method and PC-SIDE software (column 2). The prevalence of inadequate intake of Russian children in both time periods was high (23-36\%). It was much lower among US children in 1996 (2-11\%). Among the Russian girls, the prevalence increased $(\sim 13 \%)$, but among boys it decreased by a fifth over 4 years.

For all sample groups, the observed prevalence estimate was considerably higher when the (unadjusted) single day's intake was used. The bias was especially high among the US groups, $\sim 300 \%$ higher among girls (32 vs. 11\%) and $\sim 1300 \%$ among boys ( 27 vs. $2 \%$ ). Among Russian girls in 1996 , the bias was nearly $100 \%$ ( $45 \%$ vs. $23 \%$ ), among boys the prevalence was overestimated by 140\%. In 2000 the bias was similarly high (column 3).

If we had only a single day's intake for the Russian girls in 1996, and imported the variance estimates from the CSFII girls in 1996, the resulting estimate would overestimate the true value by $17 \%$ ( 27 vs. $23 \%$ ) (column 4 ). If we used the variance estimate from the RLMS 2000 girls, the results are identical to the correct value (23\%) (column 5). If we did the same for the boys, use of either the CSFII
1996 or the RLMS 2000 estimates for external variance underestimates the prevalence of inadequate intake of vitamin $\mathrm{C}$ by three percentage points, or $6 \%$.

Table 5 shows the top sources of vitamin $C$ for each sample. Russian children in both time periods obtained vitamin $\mathrm{C}$ from whole foods. The main source of vitamin C among US children was almost equally from fruit juice, fruit-flavoured beverages, and fruits and vegetables.

\section{Discussion}

The aim of this study was to examine the utility of external variance estimates to adjust the usual intake distribution of an example nutrient, vitamin C, in a group of Russian girls and boys in 1996. The results suggest that by ignoring within-person variation and applying the EAR cut-point method to a single dietary recall, the resulting prevalence estimate of inadequate nutrient intake is considerably inflated. When the intake distribution is adjusted using an external variance estimate from a different population, the prevalence estimate is much less biased, suggesting that any adjustment may give less biased estimates than no adjustment. The present study is the first to assess the possibility of using variance estimates of nutrient intake derived from one group of children to adjust the usual intake distribution and assess the prevalence of inadequate intake in another group of children. An important finding is that even though the magnitude of day-to-day variation in nutrient intake may change over

Table 5 Top sources of vitamin $C^{*}$ for sample groups, children aged $9-13$ years

\begin{tabular}{lcc}
\hline Food group & Russian children & US children \\
\hline Fruits \& vegetables & 70 & 24 \\
Mixed dishes & 14 & 13 \\
Fruit juice & 6 & 23 \\
Non-juice beverages & 4 & 24 \\
Sweets & 4 & 1 \\
Dairy products & 1 & 2 \\
Cereals & 1 & 13 \\
Total & $100 \%$ & $100 \%$ \\
\hline
\end{tabular}

${ }^{*}$ Percentage contribution. 
time in a population, when it is used to adjust or calibrate a nutrient distribution, the change may not be large enough to bias the results markedly.

Both the RLMS and the CSFII are invaluable resources for examining the effect of using external variance estimates to perform the recommended statistical adjustments to dietary data before applying the EAR cut-point method. Both are nationally representative of their respective populations and have large enough sample sizes to examine decomposed variance estimates for age and sex subgroups of children. The diet collection methodology was similar, as the RLMS collection methods were designed based upon the US Department of Agriculture's multiple-pass methodology. However, there are several concerns. First, it is difficult to compare two countries that are economically so different - the US is a developed country and Russia is a transitional country. Ideally, data used for external variance estimates should come from subjects with similar backgrounds whenever possible. The dataset must have an adequate estimate of both within- and between-person variance. A good external estimate of between-person variance is not sufficient if the sampling days are not randomly chosen, and good estimate of within-person variance is worthless if the reference study population is too homogeneous. This is why we recommend the use of a large, nationally representative dataset, such as CSFII, which fulfils both of these points. Our study shows that even with differences in culture and food environment, using the external estimate still gives less biased results than not adjusting at all. Using an estimate from a similar population would be more appropriate, but in the absence of an ideal dataset, use of even a non-ideal external estimate is preferable to a lack of adjustment. Systematic bias in reporting of dietary components has been found in US children, especially among overweight children ${ }^{34,35}$. There are no studies to our knowledge assessing the propensity to report correctly intake among Russian children.

We make several assumptions. One is that withinperson variance is constant across individuals, and therefore the within-person variability calculated from any two observed days of intake for an individual will equal that based on two observed days of intake for any other individual. Another is the potential for bias due to the different ages at which children self-report in each survey. Although the methods used to collect 24-hour recalls were similar for both the RLMS and the CSFII, the 2year difference in the age at which proxy report became self-report could lead to bias. A study by Bandini et al. reported that as girls age, they tend to underreport energy to a greater extent ${ }^{34}$. If we were to make the assumption that Russian children follow the same pattern as US children, and that the proxy report is more reliable than the older child's self-report, we may assume greater underreporting among the 10-12-year-old Russian children than among the 10-12-year-old US children.
However, we have found in previous research that the discrepancies in mean intake for older Russian and US children (where both groups self-report) is similar to that found with the present sample (Jahns L, et al., unpublished results).

The methodology does not allow for the uncertainty of the variance estimates. PC-SIDE does allow for sampling weights in the analysis, but the external estimates are treated as though estimated from the sample. In this study, the interpretation would not change based upon more conservative standard errors. Another potential problem is the FCTs used to calculate vitamin $\mathrm{C}$ intake. The US FCT reflects fortification levels of many commonly consumed items, but the Russian FCT may not accurately reflect the rapidly changing food supply in Russia. Most foods are not fortified at this point, but increasing imports may change the structure of the diet. For instance, sok or fruit juice is widely consumed in Russia, but the vitamin C content may vary considerably. An examination of Table 5 shows that the major food sources of vitamin $\mathrm{C}$ for Russian children are whole foods with relatively small (compared with fortified foods) levels of vitamin C, but are consumed frequently. Russian children consume fewer foods and have less dietary variety than US children (Jahns L, et al., unpublished results), and based upon previous research we are confident that the within-person variation in vitamin $\mathrm{C}$ intake is indeed much smaller in Russian children than in US children.

The sample of Russian children in 2000 is smaller than the other two samples, and was also collected differently, using three consecutive recalls rather than the two nonconsecutive recalls contributed by the other two samples. Although we controlled for the correlation between days in the analysis, the correlation is derived from published estimates based on a US sample and may not be appropriate for Russian children ${ }^{31}$. This bias may lead to smaller estimates of within-person variation, which would then lead to an overestimate of prevalence of nutrient inadequacy, which we did not see in this analysis.

It is difficult to compare this study to others, as no other study that we are aware of has examined the efficacy of using external estimates of within-person variation to adjust usual intake distributions for the purpose of correctly applying the EARs to assess prevalence of nutrient inadequacy. Chang et al. used an external variance estimate to adjust usual intake distributions but did not address or discuss the possible ramifications for doing $\mathrm{so}^{36}$. Murphy and Poos suggested using an estimate of within-person variation when only 1 day of intake data is available, but did not discuss how effective this may be in practice $^{17}$. In this study, we compare the prevalence estimates that are obtained using an external variance ratio with those that are obtained using the best available methodology and internal variance adjustments.

Appropriate statistical procedures can be applied to bridge the gap between observed short-term nutrient 
measurements and usual or habitual intake. Whenever possible, study design should include diet measurement on at least two non-consecutive days, and care should be taken that those days cover all weekdays and seasons, including at least one weekday and weekend per person, to obtain a more complete picture of an individual's within-person variation. Failing that, however, statistical procedures can be applied by using subgroup variance estimates from similar groups in readily available datasets.

Our previous research indicated that caution was needed when considering borrowing within-person variance, as we found large differences in within-person variation of nutrient intake between the Russian and US samples and within sexes (Jahns L, et al., unpublished results). However, the present research suggests that although the differences may appear great, when used with the ISU method the differences are not as important as failure to adjust at all.

Simulation studies would be needed to determine thresholds at which external estimates introduce bias. It may be possible that results from simulation studies could be used to produce a series of estimates of within-person variation that may be used reliably by researchers to adjust their nutrient intake distributions for population subgroups. Until such estimates exist, our results suggest that the use of external variance estimates represents a reasonable approach to using the EAR when the requisite 2 days of intake is not available.

\section{Conclusion}

Naïve application of the EAR cut-point method to unadjusted nutrient distributions will result in seriously biased prevalence estimates. External estimates of withinperson variance components perform well when compared with the correct adjustment calculated from multiple recalls in the same sample.

\section{Acknowledgements}

We wish to thank Gabriel Camaño-García for his invaluable assistance with PC-SIDE, Dan Blanchette for his SAS and Stata programming support, Ms Frances Dancy for administrative assistance and $\mathrm{Mr}$ Tom Swasey for graphics support. This research was supported by the National Institutes of Health (grants RO1-HD30880, RO1HD38700 and DK56350).

\section{References}

1 Dwyer J, Picciano MF, Raiten DJ. Future directions for the integrated CSFII-NHANES: What We Eat in AmericaNHANES. Journal of Nutrition 2003; 133: 576S-81S.

2 Woteki CE. Integrated NHANES: uses in national policy. Journal of Nutrition 2003; 133: 582S-4S

3 Nusser SM, Carriquiry AL, Dodd K, Fuller WA. A semiparametric transformation approach to estimating usual daily intake distributions. Journal of the American Statistical Association 1996; 91: 1440-9.

4 Basiotis PP, Welsh SO, Cronin FJ, Kelsay JL, Mertz W. Number of days of food intake records required to estimate individual and group nutrient intakes with desired confidence. Journal of Nutrition 1987; 117: 1638-41.

5 Carriquiry AL. Estimation of usual intake distributions of nutrients and foods. Journal of Nutrition 2003; 133: 601S-8S.

6 Dwyer J, Picciano MF, Raiten DJ. Estimation of usual intakes: What We Eat in America-NHANES. Journal of Nutrition 2003; 133: 609S-23S.

7 Gay C. Estimation of population distributions of habitual nutrient intake based on a short-run weighed food diary. British Journal of Nutrition 2000; 83: 287-93.

8 Guenther PM, Kott PS, Carriquiry AL. Development of an approach for estimating usual nutrient intake distributions at the population level. Journal of Nutrition 1997; 127: 1106-12.

9 Kohlmeier L, Bellach B. Exposure assessment error and its handling in nutritional epidemiology. Annual Review of Public Health 1995; 16: 43-59.

10 Willett W. Nutritional Epidemiology. New York: Oxford University Press, 1998.

11 Beaton GH, Milner J, Corey P, McGuire V, Cousins M, Stewart E, et al. Sources of variance in 24-hour dietary recall data: implications for nutrition study design and interpretation. American Journal of Clinical Nutrition 1979; 32: 2546-9.

12 Institute of Medicine. Dietary Reference Intakes. Applications in Dietary Assessment. Washington, DC: National Academy Press, 2000.

13 Arab L, Carriquiry A, Steck-Scott S, Gaudet MM. Ethnic differences in the nutrient intake adequacy of premenopausal US women: results from the Third National Health Examination Survey. Journal of the American Dietetic Association 2003; 103: 1008-14.

14 Pobocik RS, Benavente JC, Boudreau NS, Spore CL. Pregnant adolescents in Guam consume diets low in calcium and other micronutrients. Journal of the American Dietetic Association 2003; 10: 611-4.

15 Jahns L, Arab L, Carriquiry A. Dietary Reference Intakes still used incorrectly in journal articles. Journal of the American Dietetic Association 2003; 10: 1292-3.

16 Barr SI, Murphy SP, Poos MI. Interpreting and using the dietary references intakes in dietary assessment of individuals and groups. Journal of the American Dietetic Association 2002; 102: 780-8.

17 Murphy SP, Poos MI. Dietary Reference Intakes: summary of applications in dietary assessment. Public Health Nutrition 2002; 5: 843-9.

18 Murphy SP. Collection and analysis of intake data from the integrated survey. Journal of Nutrition 2003; 133: 585S-9S.

19 Popkin BM, Baturin A, Kohlmeier L, Zohoori N. Russia: monitoring nutritional change during the reform period. In: Wheelock V, ed. Implementing Dietary Guidelines for Healthy Eating. London: Blackie A\&P, 1997.

20 Zohoori N, Mroz T, Popkin BM, Glinskaya E, Lokshin M, Mancini DJ, et al. Monitoring the economic transition in the Russian Federation and its implications for the demographic crisis - the Russian Longitudinal Monitoring Survey. World Development 1998; 26: 1977-93.

21 Tippett KS, Cypel YS. Design and Operation: The Continuing Survey of Food Intakes by Individuals and the Diet and Health Knowledge Survey, 1994-96. Washington, DC: US Department of Agriculture, Agricultural Research Service, 1997.

22 Stang J, Story MT, Harnack L, Neumark-Sztainer D. Relationships between vitamin and mineral supplement use, dietary intake, and dietary adequacy among 
adolescents. Journal of the American Dietetic Association 2000; 100: 905-10.

23 Institute of Medicine, Food and Nutrition Board. Dietary Reference Intakes for Vitamin C, Vitamin E, Selenium, and Carotenoids. Washington, DC: National Academy Press, 2000.

24 Carriquiry AL. Assessing the prevalence of nutrient inadequacy. Public Health Nutrition 1999; 2: 23-33.

25 Murphy SP, Barr SI, Poos MI. Using the new Dietary Reference Intakes to assess diets: a map to the maze. Nutrition Reviews 2002; 60: 267-75.

26 Dodd KW. A Technical Guide to C-SIDE. Dietary Assessment Research Series Report 9. CARD Technical Report 96-TR32. Ames, IA: Center for Agricultural and Rural Development, Iowa State University, 1996.

27 Dodd KW. A User's Guide to C-SIDE: Software for Intake Distribution Estimation Version 1.O. CARD Technical Report 96-TR31. Ames, IA: Center for Agricultural and Rural Development, Iowa State University, 1996.

28 Hoffmann K, Boeing H, Dufour A, Volatier JL, Telman J, Virtanen $\mathrm{M}$, et al. Estimating the distribution of usual dietary intake by short-term measurements. European Journal of Clinical Nutrition 2002; 56(Suppl. 2): S53-62.

29 Andrews M, Kantor LS, Lino M, Ripplinger D. Using USDA's Thrifty Food Plan to assess food availability and affordability. Food Review 2001; 24: 45-53.
30 Lokshin M, Popkin BM. The emerging underclass in the Russian Federation: income dynamics 1992-96. Economic Development and Cultural Change 1999; 47: 803-29.

31 Carriquiry AL, Fuller WA, Goyeneche JJ, Jensen $\mathrm{HH}$. Estimated Correlations among Days for the Combined 1989-91 CSFII. Dietary Assessment Research Series Report 4. CARD Staff Report 95-SR77. Ames, IA: Center for Agricultural and Rural Development, Iowa State University, 1995.

32 SAS Institute Inc. SAS/STAT Software: Version 8.0. Cary, NC: SAS Institute Inc., 1999-2001.

33 StataCorp. Stata Statistical Software: Release 8.2. College Station, TX: StataCorp LP, 2003.

34 Bandini LG, Must A, Cyr H, Anderson SE, Spadano JL, Dietz WH. Longitudinal changes in the accuracy of reported energy intake in girls $10-15$ y of age. American Journal of Clinical Nutrition 2003; 78: 480-4.

35 Fisher JO, Johnson RK, Lindquist C, Birch LL, Goran MI Influence of body composition on the accuracy of reported energy intake in children. Obesity Research 2000; 8 597-603.

36 Chang HY, Suchindran CM, Pan WH. Using the overdispersed exponential family to estimate the distribution of usual daily intakes of people aged between 18 and 28 in Taiwan. Statistics in Medicine 2001; 20: 2337-50. 